\title{
Fast Cyclization of a Proline-Derived Self-Immolative Spacer Improves the Efficacy of Carbamate Prodrugs
}

\author{
Alberto Dal Corso, ${ }^{*[\mathrm{a}]}$ Valentina Borlandelli, ${ }^{[\mathrm{a}]}$ Cristina Corno,${ }^{[\mathrm{b}]}$ Paola Perego,${ }^{[\mathrm{b}]}$ Laura Belvisi, ${ }^{[\mathrm{a}]}$ \\ Luca Pignataro ${ }^{[a]}$ and Cesare Gennari; ${ }^{* a]}$
}

\begin{abstract}
Self-immolative (SI) spacers are sophisticated chemical constructs designed for molecular delivery or material degradation. We describe herein a (S)-2-(aminomethyl)pyrrolidine SI spacer able to release different types of anticancer drugs (possessing either a phenolic or secondary and tertiary hydroxyl groups) through a fast cyclization mechanism involving carbamate cleavage. The high efficiency of drug release obtained with this spacer was found to be beneficial for the in vitro cytotoxic activity of protease-sensitive prodrugs, as compared to a commonly used spacer of the same class. These findings expand the repertoire of degradation machineries and are instrumental for the future development of highly efficient delivery platforms.
\end{abstract}

\section{Introduction}

Self-immolative $(\mathrm{SI})$ spacers are covalent constructs capable of undergoing a spontaneous disassembly starting from a stable and inactive state, in response to specific stimuli. ${ }^{[1]}$ The growing interest in the generation of stimuli-responsive devices has led to the widespread application of SI spacers in different areas, including synthetic and analytical chemistry, $\left.{ }^{[2}\right]$ material sciences $^{[3]}$ and medicinal chemistry, especially in the context of prodrugs, antibody-drug conjugates (ADCs), and several other drug release strategies. ${ }^{[4]}$

Different types of activation stimuli can mediate the cleavage of a first chemical bond (SI spacer activation), which enables the SI spacer degradation and the release of active compounds (Figure $1 \mathrm{~A})$. The degradation process, driven by a positive reaction entropy, takes place through intramolecular reactions, mainly electronic cascade in aromatic or $\pi$-extended systems ${ }^{[5]}$ or cyclization of nucleophilic species (e.g. amine ${ }^{[6]}$ phenol $^{[7]}$ and thiol $^{[8]}$ groups, Figure 1B).

Carbamates have been often exploited to connect SI spacers to the active compound of interest, due to their high hydrolytic stability prior to activation and efficient cleavage via different intramolecular mechanisms. ${ }^{[9]}$ In particular, the formation of a carbamate between a hydroxyl group and an ethylenediamine fragment is by far the most exploited strategy for the release of hydroxyl-bearing payloads. ${ }^{[10]}$ In the resulting ethylenediaminecarbamate SI spacer (EthCarb, Figure 1B), the intramolecular nucleophilic attack of the amine cleaves the carbamate by forming a 5-membered cyclic urea and releases the hydroxyl

\footnotetext{
[a] Dr. A. Dal Corso, V. Borlandelli, Prof. Dr. L. Pignataro, Prof. Dr. L. Belvisi, Prof. Dr. C. Gennari

Università degli Studi di Milano, Dipartimento di Chimica

via C. Golgi, 19, I-20133, Milan, Italy.

Tel: +39 0250314091 ; Fax: +39 0250314072

E-mail: alberto.dalcorso@unimi.it; cesare.gennari@unimi.it

[b] Dr. C. Corno, Dr. P. Perego

Fondazione IRCCS Istituto Nazionale dei Tumori, Molecular Pharmacology Unit, Department of Applied Research and Technological Development, via Amadeo 42, Milan, 20133, Italy.
}

compound. The EthCarb SI spacer has shown excellent versatility, and it has been used to release bioactive molecules upon several different stimuli (e.g. enzymatic action, ${ }^{[11]}$ hypoxic conditions $^{[12]}$ and irradiation with $\mathrm{UV}^{[13]}$ or near-infrared ${ }^{[14]}$ light), while showing high stability prior to activation.

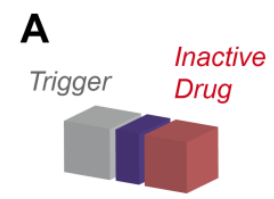

SI Spacer

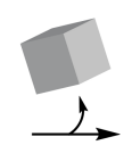

SI Spacer activation

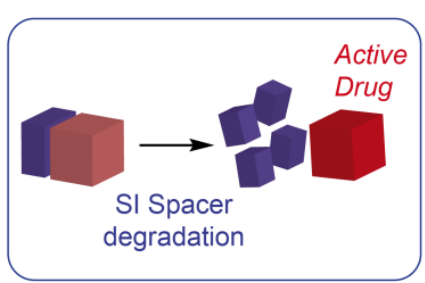

B

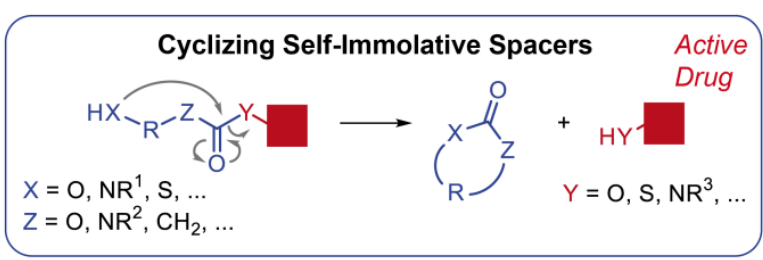

1) Ethylenediamine-Carbamate (EthCarb) Spacer

- $>300$ articles and patents

- Suboptimal release efficacy

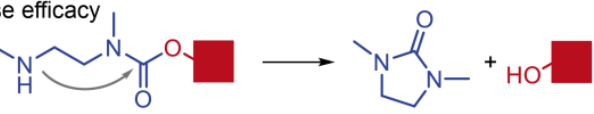

2) This Work: Proline-derived Spacer

- accelerated drug release ( $>10$-fold drop of cyclization $t_{1 / 2}$ ) - Improved anticancer efficacy in vitro

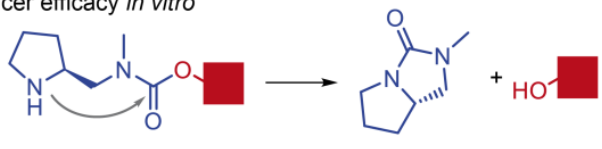

Figure 1. A) Schematic representation of the release of an active ingredient (e.g. a drug or chemical probe) upon initial activation and subsequent degradation of a self-immolative (SI) spacer. B) Mechanism of action of cyclizing SI spacers, alongside with the molecular structure of the well-known ethylenediamine-carbamate (EthCarb) spacer (1) and the proline-derived analogue (2, this work)

Noteworthy, the EthCarb spacer is connected to the phenolic $\mathrm{OH}$ group of the anticancer drug duocarmycin in the ADC [vic]trastuzumab duocarmazine (SYD985), which is currently undergoing Phase III TULIP ${ }^{\circledR}$ clinical trial with breast cancer patients. ${ }^{[15]}$ Moreover, given the promising in vivo therapeutic activity displayed in preclinical settings by other ADCs with hydroxyl-bearing (non-phenolic) payloads, ${ }^{[16]}$ the use of the EthCarb spacer could be envisaged also for the release of these drugs, even though sluggish cyclization rates have been often reported in these cases. ${ }^{[17]}$ Since slow cyclization rates may affect the therapeutic efficacy of prodrugs, a few recent examples of alternative spacers have been proposed for the release of hydroxyl-bearing (non-phenolic) payloads, in which the rate-limiting cyclization step is replaced by other cleavage mechanisms (e.g. enzymatic action ${ }^{[8]}$ or hemiaminal 
hydrolysis $\left.{ }^{[19]}\right)$. We hypothesized that suitable modifications of the ethylenediamine-carbamate module could lead to $2^{\text {nd }}$-generation cyclizing SI spacers possessing a superior drug release efficiency, while retaining the excellent versatility and stability of the traditional EthCarb spacer. This paper accounts for our efforts in this direction.

\section{Results and Discussion}

In a preliminary screening, we compared the cyclization rates of different diamine-carbamate SI spacers (e.g. Sp1-5 in Figure 2A, other spacers Sp6-8 are described in the Supporting Information, Figure $\mathrm{S} 1$ page 40) for the release of the phenolic $\mathrm{OH}$ group of the anticancer drug 7-ethyl-10-hydroxycamptothecin (SN38, Figure 2A). The synthesis of these 'spacer-SN38' prodrugs is reported in the Supporting Information. The prodrugs were isolated either as TFA-salts (Sp1-SN38, Sp3-SN38 - Sp5-SN38 in Figure 2A) or as $\mathrm{N}$-acetamides (Sp2-SN38 in Figure 2A) of their secondary amino group. These compounds were dissolved in a DMSO/acetate buffer mixture $(\mathrm{pH}=5.5)$ and incubated at $37^{\circ} \mathrm{C} \cdot{ }^{[20]}$ Aliquots were collected at different time points and the release of free SN38 was monitored by HPLC (see example in Figure 2B, complete HPLC traces are included in the Supporting Information). The percent of intact prodrug calculated from peak integrals was plotted versus time, and the cyclization rates of $\mathrm{SI}$ spacers were estimated in terms of prodrug half-life $\left(t_{1 / 2}\right)$.
A

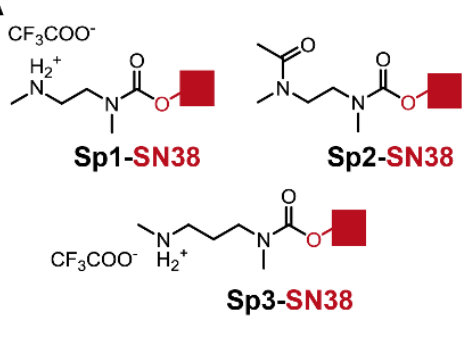<smiles>CN(CC1NCCC1OC(F)(F)F)C(=O)O[Na]</smiles><smiles>CCc1c2c(nc3ccc(O)cc13)-c1cc3c(c(=O)n1C2)COC(=O)[C@]3(O)CC1CCCCC1</smiles>

B

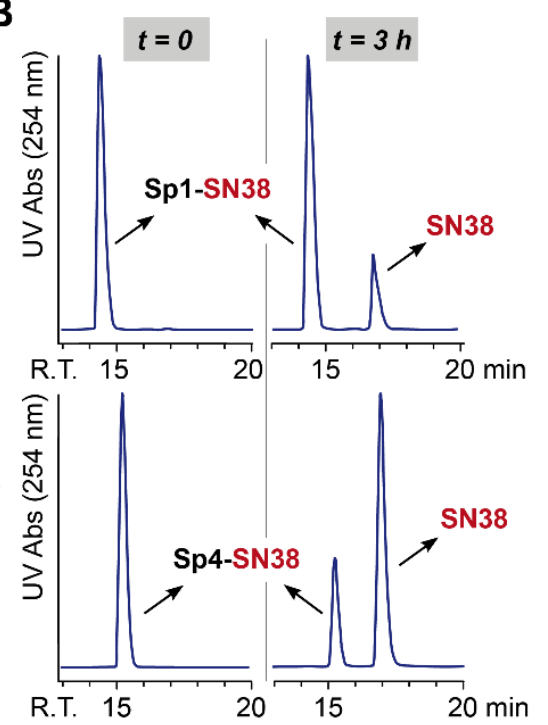

C

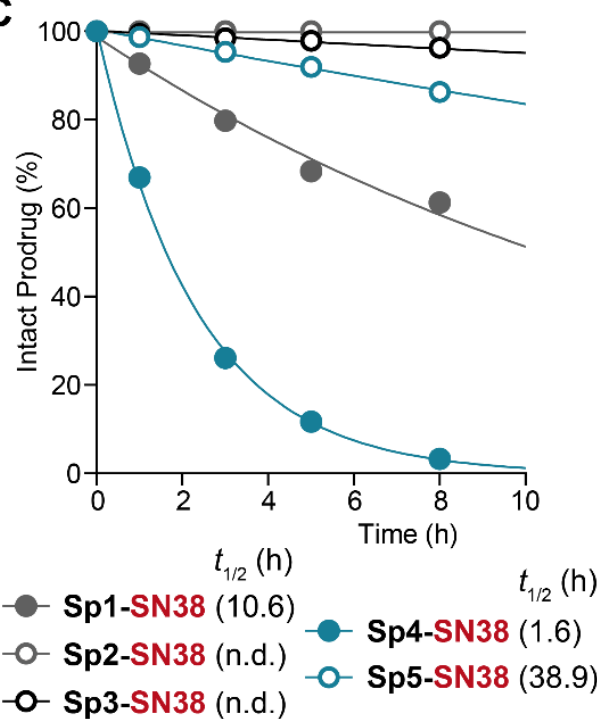

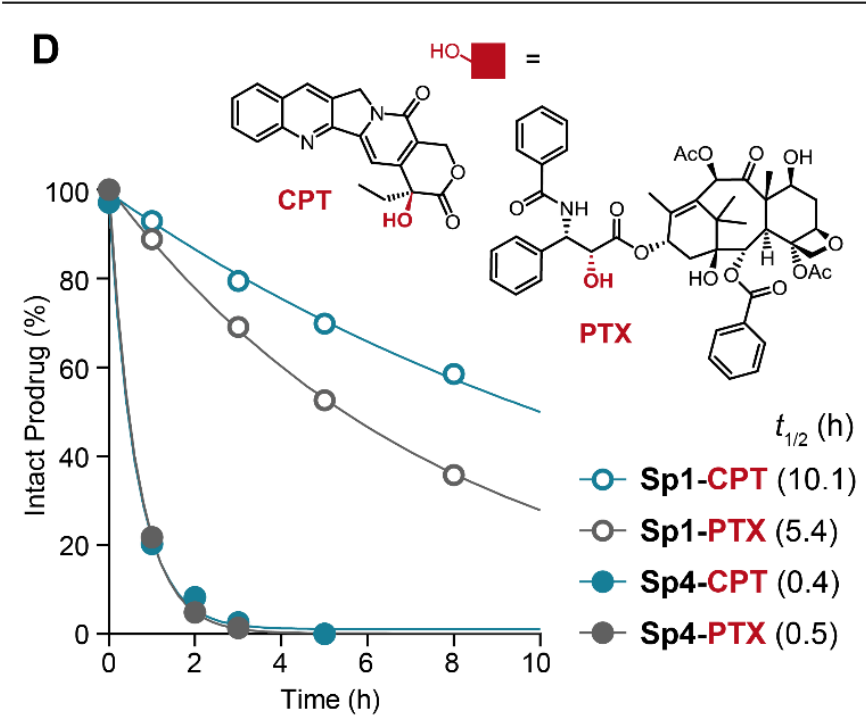

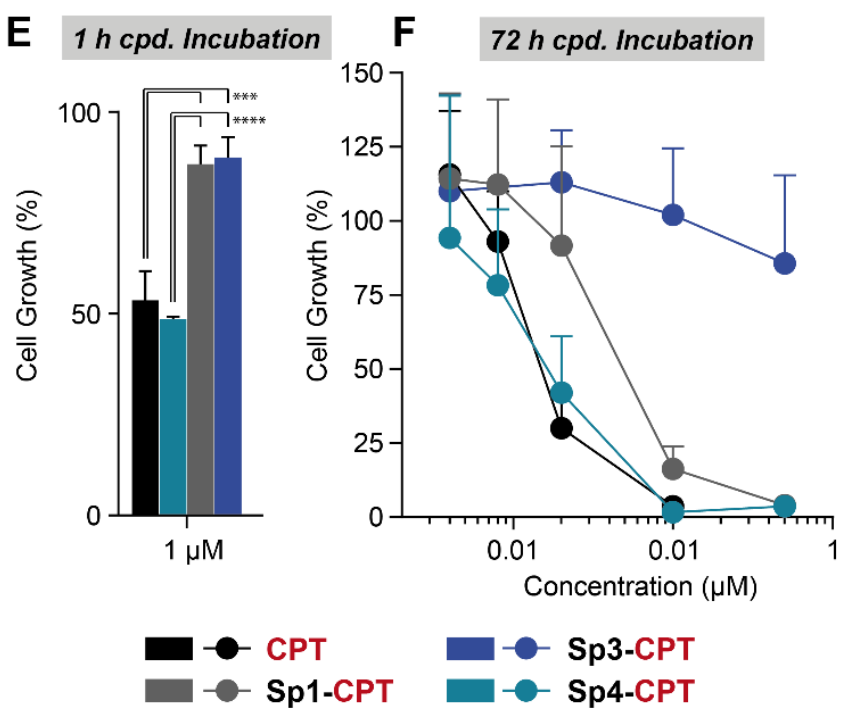

Figure 2. Molecular structure, drug release and antiproliferative activity of prodrugs bearing cyclizing SI spacers. A) Structure of diamine-carbamate SI spacers Sp1-5, connected to the phenolic O atom of anticancer drug 7-ethyl-10-hydroxycamptothecin (SN38) via a carbamate. B) Example of HPLC traces for the stability analysis of prodrugs Sp1-SN38 and Sp4-SN38 and estimation of drug release efficacy: compounds were incubated at $37{ }^{\circ} \mathrm{C}$ in a DMSO/acetate buffer mixture $(\mathrm{pH}=5.5)$ and the decay of the HPLC peak corresponding to the initial prodrug was monitored over time (complete HPLC traces are included in the Supporting Information). C) Summary of the stability analysis of the 5 prodrugs for the release of SN38 at $37{ }^{\circ} \mathrm{C}$ and $\mathrm{pH}=5.5$ (measured $t_{1 / 2}$ are reported in brackets). D) Summary of the stability analysis of paclitaxel (PTX) and camptothecin (CPT) prodrugs featuring the cyclizing spacers Sp1 and $\mathrm{Sp} 4$ at $37{ }^{\circ} \mathrm{C}$ and $\mathrm{pH}=7.5$ (measured $t_{1 / 2}$ are reported in brackets). E) Cell growth inhibition assays of ovarian carcinoma (IGROV-1) cells upon 1-hour incubation with prodrugs Sp1-CPT, Sp3-CPT, Sp4-CPT and free CPT at $1 \mu \mathrm{M}$ concentration, followed by cell washout and incubation for additional $72 \mathrm{~h}$ in fresh medium. The experiment was performed 3 times in duplicates, error bars represent $\mathrm{SD},{ }^{* * *} p<0.001,{ }^{* * *} p<0.0001$. F) Cell growth inhibition assays of IGROV-1 cells upon 72 -hour incubation with serial dilutions of prodrugs Sp1-CPT, Sp3-CPT, Sp4-CPT and free CPT. IC $50 \sim 10 \mathrm{nM}(\mathrm{CPT}), \mathrm{IC}_{50} \sim 50 \mathrm{nM}$ (Sp1-CPT), IC $50>500 \mathrm{nM}(\mathrm{Sp3}-\mathrm{CPT}), \mathrm{IC}_{50} \sim 20 \mathrm{nM}$ (Sp4-CPT), as estimated by linear regression. The experiment was performed 3 times in duplicates, error bars represent SD. 
As shown in Figure 2C, the EthCarb spacer in prodrug Sp1SN38 showed $t_{1 / 2}=10.6 \mathrm{~h}$ (consistent with literature data), ${ }^{[21]}$ whereas the inertness of the acetylated prodrug Sp2-SN38 indicates that the carbamate between the spacer and the drug is not hydrolyzed under these experimental conditions. Prodrug Sp3-SN38 - endowed with a propanediamine-carbamate SI spacer cyclizing to the corresponding six-membered cyclic urea (Sp3-U in Figure 3B) - gave much slower drug release than Sp1-SN38, which forms a five-membered urea (Sp1-U in Figure 3B). Such a different behavior is consistent with the generally higher rate of cyclization reactions forming five-membered rings compared to those affording six-membered rings. ${ }^{[22]}$ Among all tested prodrugs, Sp4-SN38 was found to release the free drug with the highest rate $\left(t_{1 / 2}=1.6 \mathrm{~h}\right) .{ }^{[23]}$ In this prodrug, a carbamate connects the drug's phenolic oxygen to the exocyclic $N$ atom of (S)-2-(aminomethyl)pyrrolidine, and drug release occurs through nucleophilic attack of the endocyclic amino group to the carbamate bond and formation of the corresponding bicyclic urea (Sp4-U in Figure 3B). ${ }^{[2]}$ Interestingly, drug conjugation at the endocyclic $N$ atom of (S)-2-(aminomethyl)pyrrolidine was found to be detrimental for the spacer efficiency, as prodrug Sp5-SN38 (Figure $2 \mathrm{~A})$ gave slow drug release $\left(t_{1 / 2}=38.9 \mathrm{~h}\right.$ ).

To explore the efficiency of the best-performing Sp4 spacer for the release of anticancer drugs possessing non-phenolic hydroxyl groups, this SI spacer was conjugated to the secondary $\mathrm{OH}$ group at the 2'-position of paclitaxel (PTX) and to the tertiary $\mathrm{OH}$ group of camptothecin (CPT). The drug release efficiency of the resulting prodrugs Sp4-PTX and Sp4-CPT was evaluated as described above, in parallel with the EthCarb-bearing analogues Sp1-PTX and Sp1-CPT (Figure 2D). In all cases, prodrug incubation at acidic $\mathrm{pH}$ (5.5) did not lead to significant drug release within 8 hours (see the Supporting Information, pages 36 and 39). On the other hand, when prodrugs were incubated in a DMSO/phosphate buffer mixture $(\mathrm{pH}=7.5)$ at $37^{\circ} \mathrm{C}$, the Sp4 spacer was found to release both free PTX and CPT at much higher rates $\left(t_{1 / 2} \sim 0.5 \mathrm{~h}\right.$ measured for both prodrugs) than the traditional EthCarb spacer $\left(t_{1 / 2}=5.4\right.$ and $10.1 \mathrm{~h}$ for Sp1-PTX and Sp1-CPT, respectively).

Overall, these data indicate that the SI spacer Sp4 is much more efficient than the commonly used Sp1. Interestingly, no previous literature data correlate the different release efficiency of this class of SI spacers with the effective anticancer properties of the different prodrugs. To investigate this aspect, the ovarian carcinoma cell line IGROV-1 was treated with free CPT and with three CPT prodrugs possessing different cyclizing SI spacers (Sp1-CPT, Sp3-CPT and Sp4-CPT), dissolved in the cell medium at $1 \mu \mathrm{M}$ concentration. After incubation for 1 hour, cells were washed and then incubated for 72 hours in fresh medium, followed by quantitative analysis of cell growth. This limited cell exposure to the prodrug mimics the typical conditions of extracellular drug delivery in vivo, ${ }^{[25]}$ in which CPT is released from a carrier in the tumor microenvironment and then the fundamental steps for its cytotoxic activity (i.e. diffusion through the cell membrane and inhibition of the intracellular target, i.e. DNA topoisomerase I) compete with the drug clearance from the tumor tissue. As can be seen in the histogram in Figure 2E, the anticancer activity of the Sp4-CPT prodrug under these experimental conditions was comparable to that of free CPT (i.e. cell growth reduced to ca. $50 \%$ compared to untreated cells), whereas prodrug Sp1-CPT showed lower anticancer activity, similar to the one displayed by the slow-cyclizing prodrug Sp3-
CPT. The experiment was repeated with a cell growth inhibition assay with long-term drug exposure, and cells were exposed to the tested compounds for 72 hours (Figure 2F). Also in this case, Sp4-CPT displayed a growth inhibitory activity very similar to that of free CPT. Interestingly, Sp1-CPT proved more active than Sp3-CPT under these experimental conditions, but its lower activity compared to Sp4-CPT indicates that a rapid SI spacer degradation is fundamental to achieve significant cell killing even when cancer cells are exposed to the prodrugs for long periods of time.

Following these proof-of-concept experiments, we investigated the possibility to incorporate the Sp4 spacer in model prodrugs, endowed with a generic trigger to achieve selective drug release at the tumor site. Following a typical design of linker-drug modules in ADCs, ${ }^{[15,16]}$ three CPT prodrugs (compounds 1a-3a in Figure $3 \mathrm{~A}$ ) and three PTX prodrugs (compounds $\mathbf{1 b} \mathbf{b} \mathbf{3 b}$ in Figure $3 \mathrm{~A}$ ) were synthesized, featuring a peptide trigger (i.e. the Val-Arg dipeptide) prone to rapid proteolytic cleavage under standard cell culture conditions. ${ }^{[26]}$ The Val-Arg trigger was functionalized at the C-terminus with the well-known electronic cascade spacer para-aminobenzyl carbamate (PABC) and with a hydrophilic moiety at the $\mathrm{N}$ terminus, which improves the prodrug solubility in aqueous media.

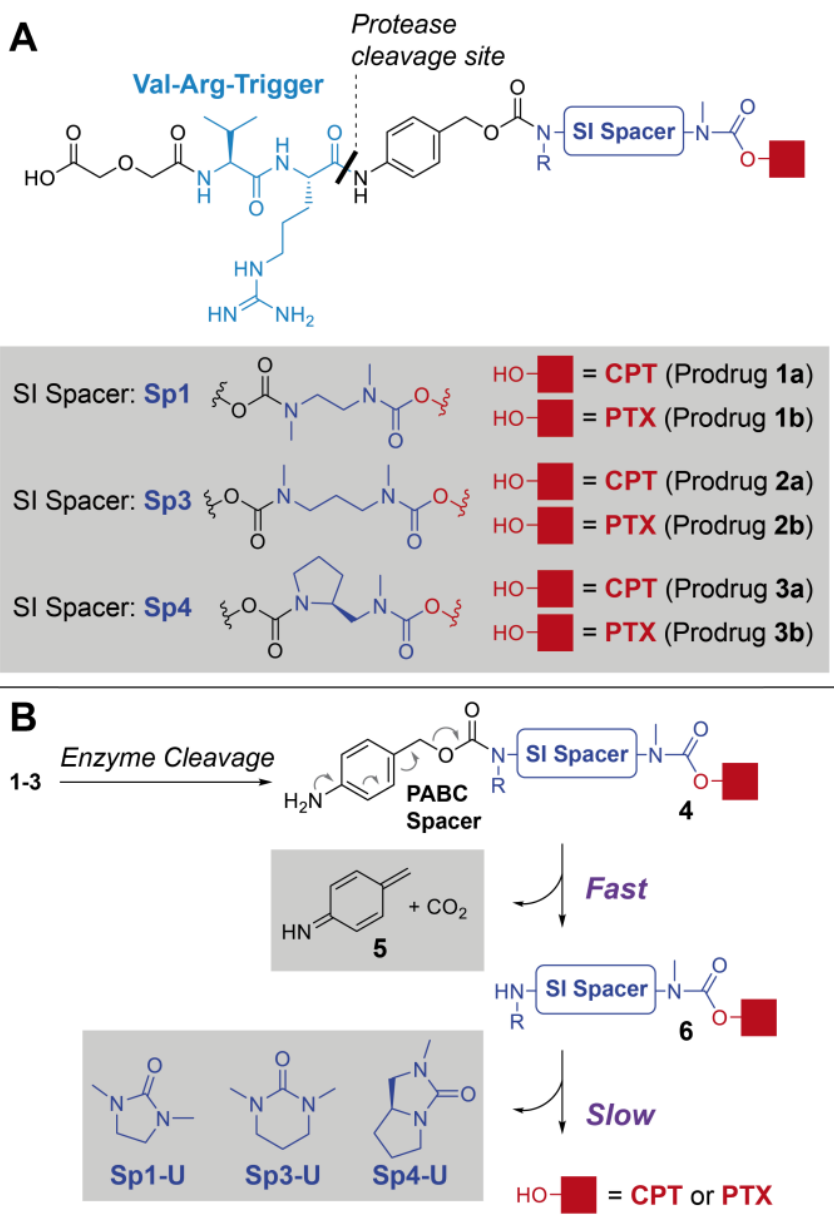

Figure 3. A) Molecular structure of prodrugs 1-3, featuring a payload [camptothecin (CPT) or paclitaxel (PTX)], a protease-cleavable dipeptide trigger (Val-Arg), a para-aminobenzyl carbamate (PABC) spacer and three different cyclizing SI spacers. B) Mechanism of action of prodrugs 1-3 for payload release and molecular structures of the 3 different cyclic urea products derived from the spacer cyclization (Sp1-U, Sp3-U and Sp4-U). 
Table 1. Cell growth inhibition assays of IGROV-1 cells upon incubation with prodrugs 1-3 and free payloads CPT and PTX. ${ }^{[\mathrm{a}]}$

\begin{tabular}{cll}
\hline Compound & & IC $_{50}[\mathrm{~nm}]^{[\mathrm{b}]}$ \\
\hline CPT & Camptothecin & $24.0 \pm 8.7$ \\
1a & Val-Arg-PABC-Sp1-CPT & $73.9 \pm 28.5$ \\
2a & Val-Arg-PABC-Sp3-CPT & $860.6 \pm 674.0$ \\
3a & Val-Arg-PABC-Sp4-CPT & $30.2 \pm 10.3$ \\
\hline Compound & & IC $_{50}[\boldsymbol{\mu m}]^{[\mathrm{cc}]}$ \\
\hline PTX & Paclitaxel & $0.07 \pm 0.03$ \\
1b & Val-Arg-PABC-Sp1-PTX & $1.44 \pm 0.72$ \\
2b & Val-Arg-PABC-Sp3-PTX & n.d. ${ }^{[\mathrm{d}]}$ \\
3b & Val-Arg-PABC-Sp4- PTX & $0.79 \pm 0.23$ \\
\hline
\end{tabular}

[a] All assays were performed 3 times (three independent experiments) in duplicates. $\mathrm{IC}_{50}$ values were estimated by linear regression, as described in the Supporting Information, page 40. [b] Cells were incubated for 72 hours with serial dilutions of prodrugs 1a-3a and free CPT. [c] Cells were incubated for 24 hours with serial dilutions of prodrugs $1 \mathbf{b}-\mathbf{3 b}$ and free PTX, followed by cell washout and incubation for additional $48 \mathrm{~h}$ in fresh medium. [d] n.d.: not determined $\left(\mathrm{IC}_{50}>>10 \mu \mathrm{M}\right)$.

Compounds 1, 2 and 3 possess different cyclization SI spacers (Sp1, Sp3 and Sp4, respectively), which connect the payload and the PABC spacer through two cleavable carbamate bonds. Figure $3 \mathrm{~B}$ illustrates the drug release mechanism of prodrugs 13: the dipeptide cleavage in the presence of proteases results in the initial formation of the aniline metabolite $\mathbf{4}$, which is rapidly converted to the azaquinone intermediate 5 , releasing $\mathrm{CO}_{2}$ and the amine-prodrugs 6 . Finally, payload release occurs through cyclization of the amine-prodrugs, which typically represents the rate-limiting step of the whole drug release process. ${ }^{[10]}$

Similarly to the antiproliferative assays described above for the amine-prodrugs (Figure 2F), IGROV-1 cancer cells were incubated for $72 \mathrm{~h}$ with decreasing concentrations of prodrugs 1$3^{[27]}$ The cytotoxic activity of compounds 1-3 (data reported in Table 1 and Figure S4, page 42 of the Supporting Information) reflected the cyclization reactivity of the different SI spacers: prodrug 3 (SI spacer: Sp4) proved more potent than prodrug 1 (SI spacer: Sp1), while prodrug 2 (SI spacer: Sp3) resulted practically inactive. In particular, the protease-activable CPT prodrug 3a, featuring the fast-cyclizing SI spacer Sp4, exhibited a cytotoxic activity similar to free CPT $\left(3 a, I_{50}=30.2 \mathrm{nM}\right.$; CPT, $\left.\mathrm{IC}_{50}=24.0 \mathrm{nM}\right)$. In the PTX series, prodrug $3 \mathrm{~b}$ proved less potent than free PTX by one order of magnitude $\left(\mathbf{3 b}, \mathrm{IC}_{50}=0.79\right.$ $\mu \mathrm{M}$; PTX, $\mathrm{IC}_{50}=0.07 \mu \mathrm{M}$ ) but still appreciably more active than prodrug $\mathbf{1 b}$ featuring the traditional EthCarb spacer $\left(\mathbf{1} \mathbf{b}, \mathrm{IC}_{50}=\right.$ $1.44 \mu \mathrm{M})$.

\section{Conclusion}

This work demonstrates that the cyclization efficiency of SI spacers strongly impacts on the anticancer potency of model prodrugs. Kinetic studies on drug release and in vitro studies in IGROV-1 cancer cells showed that not only the proline-derived SI spacer Sp4 can release hydroxy-bearing payloads with faster rates than the commonly used analog Sp1, but also that this spacer can improve the cell-killing activity of anticancer prodrugs.
Unexpectedly, the superior biological activity of Sp4 compared to Sp1 was detectable also upon long term exposure of cancer cells to the prodrugs (72 hours). This observation indicates that fast SI spacers are fundamental to quickly reach high concentrations of active compound in the tumor environment, which may prevent the development of drug resistance and the proliferation of resilient cells. ${ }^{[28]}$ These in vitro data hold promise for the installation of the Sp4 spacer in a large subset of activable platforms for in vivo therapy and diagnosis. The rate of self-immolative cleavage is expected to be particularly relevant in the context of non-internalizing conjugates. ${ }^{[2]}$ In fact, in this case the therapeutic outcome is a composite result of efficient targeting, rate of linker cleavage (SI spacer activation) and rate of SI spacer degradation, which may regulate the competition between drug entry into the cells and drug escape back into the bloodstream.

\section{Experimental Section}

All synthetic procedures (along with the ${ }^{1} \mathrm{H}$ - and ${ }^{13} \mathrm{C}-\mathrm{NMR}$ spectra, HPLC and MS data) and all procedures for biochemical and biological assays are included in the Supporting Information.

\section{Acknowledgements}

We thank T. Zaninelli, M. Bottanelli and M. Frigoli for their contribution to the prodrug synthesis. We gratefully acknowledge Ministero dell'Università e della Ricerca (PRIN 2015 project 20157WW5EH) for financial support.

Keywords: Cascade Reactions • Disassembly • Drug delivery • Prodrugs $\cdot$ Self-Immolative Spacers

\section{References}

[1] A. Alouane, R. Labruère, T. Le Saux, F. Schmidt, L. Jullien, Angew. Chem. Int. Ed. 2015, 54, 7492-7509; Angew. Chem. 2015, 127, 7600 7619 .

[2] a) Y. K. Qi, S. Tang, Y. C. Huang, M. Pan, J. S. Zheng, L. Liu, Org Biomol. Chem. 2016, 14, 4194-4198; b) M. E. Roth-Konforti, C. R. Bauer D. Shabat, Angew. Chem. Int. Ed. 2017, 56, 15633-15638; Angew. Chem 2017, 129, 15839-15844; c) M. E. Roth, O. Green, S. Gnaim, D. Shabat Chem. Rev. 2016, 116, 1309-1352.

[3] a) W. Seo, S. T. Phillips, J. Am. Chem. Soc. 2010, 132, 9234-9235; b) R A. McBride, E. R. Gillies, Macromolecules 2013, 46, 5157-5166; c) S Gnaim, D. Shabat, J. Am. Chem. Soc. 2017, 139, 10002-10008.

[4] a) N. Krall, J. Scheuermann, D. Neri, Angew. Chem. Int. Ed. 2013, 52, 1384-1402; Angew. Chem. 2013, 125, 1424-1443; b) M. Srinivasarao, P. S. Low, Chem. Rev. 2017, 117, 12133-12164; c) R. Walther, J. Rautio, A N. Zelikin, Adv. Drug Deliv. Rev. 2017, 118, 65-77.

[5] S. Gnaim, D. Shabat, Acc. Chem. Res. 2014, 47, 2970-2984

[6] K. Haba, M. Popkov, M. Shamis, R. A. Lerner, C. F. Barbas, D. Shabat, Angew. Chem. Int. Ed. 2005, 44, 716-720; Angew. Chem. 2005, 117, 726-730.

[7] S. Huvelle, A. Alouane, T. Le Saux, L. Jullien, F. Schmidt, Org. Biomol. Chem. 2017, 15, 3435-3443.

[8] a) Y. Meyer, J. A. Richard, M. Massonneau, P. Y. Renard, A. Romieu Org. Lett. 2008, 10, 1517-1520; b) Y. Meyer, J. A. Richard, B. Delest, P. Noack, P. Y. Renard, A. Romieu, Org. Biomol. Chem. 2010, 8, 1777-1780. 
[9] A. Dal Corso, L. Pignataro, L. Belvisi, C. Gennari, Chem. Eur. J. 2019, 25, 14740-14757.

[10] a) W. S. Saari, J. E. Schwering, P. A. Lyle, S. J. Smith, E. L. Engelhardt, J Med. Chem. 1990, 33, 97-101; b) F. M. H. de Groot, L. W. A. van Berkom, H. W. Scheeren, J. Med. Chem. 2000, 43, 3093-3102; c) M. Shamis, H. N. Lode, D. Shabat, J. Am. Chem. Soc. 2004, 126, 1726-1731; d) E. KisinFinfer, S. Ferber, R. Blau, R. Satchi-Fainaro, D. Shabat, Bioorg. Med. Chem. Lett. 2014, 24, 2453-2458.

[11] a) X. Zhang, K. Tang, H. Wang, Y. Liu, B. Bao, Y. Fang, X. Zhang, W. Lu, Bioconjugate Chem. 2016, 27, 1267-1275; b) B. Bao, Y. Liu, L. Wang, W. Lu, RSC Adv. 2016, 6, 69540-69545; c) E. Bouvier, S. Thirot, F. Schmidt, C. Monneret, Org. Biomol. Chem. 2003, 1, 3343-3352; d) S. C. Jeffrey, J. De Brabander, J. Miyamoto, P. D. Senter, ACS Med. Chem. Lett. 2010, 1 277-280; e) B. Huang, A. Desai, S. Tang, T. P. Thomas, J. R. Baker Jr., Org. Lett. 2010, 12, 1384-1387; f) A. Dal Corso, M. Caruso, L. Belvisi, D. Arosio, U. Piarulli, C. Albanese, F. Gasparri, A. Marsiglio, F. Sola, S. Troiani, B. Valsasina, L. Pignataro, D. Donati, C. Gennari, Chem. Eur. J. 2015, 21, 6921-6929.

[12] C. Jin, Q. Zhang, W. Lu, Eur. J. Med. Chem. 2017, 132, 135-141.

[13] a) P.T. Wong, E. W. Roberts, S. Tang, J. Mukherjee, J. Cannon, A. J. Nip, K. Corbin, M. F. Krummel, S. K. Choi, ACS Chem. Biol. 2017, 12, 1001-1010; b) S. Shah, P. K. Sasmal, K. B. Lee, J. Mater. Chem. B. 2014 2, 7685-7693; c) M. J. O'Connor, L. L. Beebe, D. Deodato, R. E. Ball, A. T. Page, A. J. VanLeuven, K. T. Harris, S. Park, V. Hariharan, J. D. Lauderdale, T. M. Dore, ACS Chem. Neurosci. 2019, 10, 266-278; d) N. Fomina, C. L. McFearin, A. Almutairi, Chem. Commun. 2012, 48, 91389140.

[14] a) A. P. Gorka, R. R. Nani, J. Zhu, S. Mackem, M. J. Schnermann, J. Am. Chem. Soc. 2014, 136, 14153-14159; b) Q. Miao, D. C. Yeo, C. Wiraja, J. Zhang, X. Ning, C. Xu, K. Pu, Angew. Chem. Int. Ed. 2018, 57, 12561260; Angew. Chem. 2018, 130, 1270-1274.

[15] a) R. C. Elgersma, R. G. Coumans, T. Huijbregts, W. M. Menge, J. A. Joosten, H. J. Spijker, F. M. de Groot, M. M. van der Lee, R. Ubink, D. J. van den Dobbelsteen, D. F. Egging, W. H. Dokter, G. F. Verheijden, J. M. Lemmens, C. M. Timmers, P. H. Beusker, Mol. Pharm. 2015, 12, 1813 1835; b) P. H. Beusker, R. G. Coumans, R. C. Elgersma, W. M. Menge, J A. Joosten, H. J. Spijker, F. M. de Groot, (Synton), PCT/NL2011/050278, WO/2011/133039, 2011.

[16] a) S. F. Yu, B. Zheng, M. Go, J. Lau, S. Spencer, H. Raab, R. Soriano, S Jhunjhunwala, R. Cohen, M. Caruso, P. Polakis, J. Flygare, A. G. Polson, Clin. Cancer Res. 2015, 21, 3298-3306; b) A. Dal Corso, R. Gébleux, P. Murer, A. Soltermann, D. Neri, J. Controlled Release 2017, 264, 211-218.

[17] A. Raposo Moreira Dias, A. Pina, A. Dean, H. G. Lerchen, M. Caruso, F. Gasparri, I. Fraietta, S. Troiani, D. Arosio, L. Belvisi, L. Pignataro, A. Dal Corso, C. Gennari, Chem. Eur. J. 2019, 25, 1696-1700.

[18] J. C. Kern, D. Dooney, R. Zhang, L. Liang, P. E. Brandish, M. Cheng, G. Feng, A. Beck, D. Bresson, J. Firdos, D. Gately, N. Knudsen, A. Manibusan, Y. Sun, R. M. Garbaccio, Bioconjugate Chem. 2016, 27, 2081-2088.

[19] a) R. V. Kolakowski, K. T. Haelsig, K. K. Emmerton, C. I. Leiske, J. B. Miyamoto, J. H. Cochran, R. P. Lyon, P. D. Senter, S. C. Jeffrey, Angew. Chem. Int. Ed. 2016, 55, 7948-7951; Angew. Chem. 2016, 128, 8080 8083 ; b) Y. Ogitani, T. Aida, K. Hagihara, J. Yamaguchi, C. Ishii, N. Harada, M. Soma, H. Okamoto, M. Oitate, S. Arakawa, T. Hirai, R. Atsumi, T. Nakada, I. Hayakawa, Y. Abe, T. Agatsuma, Clin. Cancer Res. 2016, 22, 5097-5108.

[20] A slightly acidic pH (5.5) was selected for this screening because diamine-carbamate SI spacers release phenolic compounds too rapidly at higher $\mathrm{pH}$ values. For example, prodrug Sp1-SN38 showed $t_{1 / 2}$ for the release of $\mathrm{SN} 38=10.6 \mathrm{~h}$ at $\mathrm{pH} 5.5$ and $\sim 1 \mathrm{~min}$ at $\mathrm{pH} 7.5$, see the Supporting Information page 25 . The slower release rates detected at $\mathrm{pH}$ 5.5 facilitate the comparative analysis shown in Figure 2C.

[21] S. V. Govindan, T. M. Cardillo, R. M. Sharkey, F. Tat, D. V. Gold, D. M. Goldenberg, Mol. Cancer. Ther. 2013, 12, 968-978.

[22] A. C. Knipe, C. J. Stirling, J. Chem. Soc. B. 1968, 67-71.

[23] The drug release properties of Sp4 may be reminiscent of the conformational preferences of peptide sequences which include proline residues (e.g. the Gly-Pro dipeptide), that give rise, in peptides and proteins, to the formation of specific secondary structures (e.g. $\beta$-turns) or post-translational modifications (e.g. formation of diketopiperazines) Diketopiperazine formation was also investigated for drug delivery purposes, see: a) S. Manabe, H. Machida, Y. Aihara, M. Yasunaga, Y Itoa, Y. Matsumura, Med. Chem. Commun. 2013, 4, 792-796; b) E. Figueras, A. Martins, A. Borbély, V. Le Joncour, P. Cordella, R. Perego, D. Modena, P. Pagani, S. Esposito, G. Auciello, M. Frese, P. Gallinari, P. Laakkonen, C. Steinkühler, N. Sewald, Pharmaceutics 2019, 11, E220.

[24] Sp4-U was detected by LC-MS analysis (see the Supporting Information). [25] a) A. H. Staudacher, M. P. Brown, Br. J. Cancer 2017, 117, 1736-1742; b) B. Renoux, F. Raes, T. Legigan, E. Peraudeau, B. Eddhif, P. Poinot, I. Tranoy-Opalinski, J. Alsarraf, O. Koniev, S. Kolodych, S. Lerondel, A. Le Pape, J. Clarhautad, S. Papot, Chem. Sci. 2017, 8, 3427-3433.

[26] S. Cazzamalli, A. Dal Corso, D. Neri, J. Controlled Release 2017, 246, 39-45.

[27] With CPT we observed the largest difference between Sp1 and Sp4 in terms of release rates (i.e. Sp4 is 25.3 times faster than Sp1 with CPT, while it is 10.8 times faster with PTX, see Figure 2D). The cell growth inhibition assays were designed in order to take into account these kinetic differences: in the experiment with CPT and prodrugs 1a-3a cells were incubated for 72 hours, while in the experiment with PTX and prodrugs 1b-3b cells were incubated for 24 hours, followed by cell washout and incubation for additional $48 \mathrm{~h}$ in fresh medium.

[28] S. Agnello, M. Brand, M. F. Chellat, S. Gazzola, R. Riedl, Angew. Chem. Int. Ed. 2019, 58, 3300-3345; Angew. Chem. 2019, 131, 3336-3383. 